\title{
Similitudes y diferencias entre Redes de Sensores Inalámbricas e Internet de las Cosas: Hacia una postura clarificadora
}

\section{Similarities and differences between Wireless Sensor Networks and the Internet of Things: Towards a clarifying position}

\author{
Johan S. Rueda R. ${ }^{1(D)}$, Jesús M. Talavera. P. ${ }^{2(D)}$ \\ ${ }^{1}$ Centro de Excelencia y Apropiación en Internet de las Cosas (CEA-IoT) Nodo Oriente, Universidad Autónoma \\ de Bucaramanga, Colombia \\ ${ }^{2}$ Laboratorio de investigación en fusión de datos, información y conocimiento de la Universidad Federal de Río \\ de Janeiro, Brasil \\ irueda526@unab.edu.co, jesus.tp@ufrj.br
}

(Recibido: 5 Abril 2017, aceptado: 20 Abril 2017)

\begin{abstract}
Resumen. Las redes de sensores inalámbricas (WSN) e Internet de las Cosas (IoT) son dos áreas de estudio que comparten entre sí ser una infraestructura de red autónoma, en la cual se interconectan objetos para medir variables físicas y dar solución a problemas en una variedad de escenarios de aplicación, como logística, industria, construcciones inteligentes, seguridad, agricultura, entre otros. Esta semejanza suscita una ambigüedad en el uso que la comunidad académica hace de los términos, WSN e IoT, y hace borrosa la línea de dónde pertenecen las contribuciones que se realizan en cada una de estas áreas. En consecuencia, el objetivo de este artículo es analizar la relación, similitud y diferencias entre WSN e IoT en torno a cinco temas: conceptos, requisitos generales, arquitecturas, aplicaciones y tratamiento de datos. A pesar de que WSN e IoT tienen un origen en común, sus enfoques son diferentes en varios aspectos que permiten aclarar la ambigüedad suscita entre la comunidad académica.
\end{abstract}

Palabras Clave: Aplicaciones distribuidas, Internet de las cosas; redes de sensores inalámbricas.

\begin{abstract}
Wireless Sensor Network (WSN) and Internet of Things (IoT) are two fields of study, which share, being an autonomous network infrastructure, where objects are interconnected to measure physical variables in scenarios such as logistics, industry, intelligent constructions, security, agriculture, among others. This similarity raises an ambiguity in the academic community's use of the terms WSN and IoT doing blurred the line of where belong the contributions that are made in each of these areas of study. Therefore, the purpose of this article is to analyze the relationship, similarity, and differences between WSN and IoT around five topics, namely: conceptual level, its general requirements and architectures, application construction and data processing. Although WSN and IoT have a common origin, their approaches are different in several ways that clarify the ambiguity arouses among the academic community.
\end{abstract}

Keywords: Distributed applications, Internet of Things; IoT; Wireless Sensor Network; WSN.

\section{Introducción}

El concepto de redes de sensores surgió en las últimas décadas del siglo XX y se conocían como redes de sensores distribuidos (DSN, por sus siglas en inglés, Distributed Sensor Network). En la década de 1980, 
se conocieron los primeros trabajos en la literatura científica sobre esta tecnología. En 1981, investigadores realizaban experimentos en laboratorio para determinar algunos principios generales de diseño de estas redes [1], así mismo, otros trabajos científicos hablaban de la agregación de datos realizada por los sensores [2, 3], y planteaban soluciones a problemas importantes de las redes de sensores distribuidas [4].

El sector de estandarización en telecomunicaciones de la International Telecommunication Union (ITU-T), define una red de sensores como una red compuesta por nodos sensores interconectados, que intercambian datos detectados, usando comunicación inalámbrica o cableada [5, 6]. Estas redes de sensores están densamente desplegadas, ya sea dentro del ambiente donde ocurre un fenómeno o muy cerca de él, y se basan en el esfuerzo colaborativo de todos sus nodos para la obtención de los datos [7].

Las redes de sensores inalámbricas (WSN, por sus siglas en inglés, Wireless Sensor Network) consisten en un conjunto de cientos a miles de dispositivos autónomos, llamados nodos sensores, que están distribuidos físicamente en un área geográfica para monitorizar condiciones físicas o ambientales, con capacidad de almacenar y comunicar datos en una red de forma inalámbrica. Un nodo sensor está compuesto por un procesador, una memoria, un transceptor, uno o más sensores, un conversor de señal análoga a digital (ADC) y una fuente de alimentación. No obstante, los nodos sensores poseen limitaciones en cuanto a: (i) alcance de transmisión; (ii) capacidad de procesamiento; (iii) capacidad de almacenamiento, y (iv) suministro de energía. Esto último, es un factor determinante al momento de estimar el tiempo de vida de la red y por ello es un factor de diseño en aplicaciones WSN.

Dadas estas limitaciones, las investigaciones sobre WSN centran sus esfuerzos en estudiar y generar soluciones para que los nodos sensores hagan uso óptimo de sus recursos, como, por ejemplo, uso de la energía $[8,9]$, comunicación de corto alcance $[10,11]$ y conectividad entre nodos sensores [12]. De este tipo de investigaciones nacieron protocolos y tecnologías de comunicación que optimizan el uso de energía como LoRaWAN [13] y ZigBee [14], este último basado en el estándar IEEE 802.15.4 [15].

Otra estrategia/solución para la optimización de recursos, es la implementación de protocolos eficientes de enrutamiento en las WSN. Estos protocolos son los responsables de mantener las rutas en la red y tienen que garantizar la fiabilidad de la comunicación en múltiples saltos, considerando las condiciones de hardware de los nodos sensores. Entre los retos y los problemas de enrutamiento que deben considerar estos protocolos son las limitaciones de recursos en términos de energía y eficiencia del hardware, el despliegue masivo de los nodos sensores, las características de la red y del medio ambiente, y la agregación de datos [16].

En lo relativo al despliegue de las redes de sensores inalámbricas, se pueden considerar tres tipos de implantación: (i) determinística, (ii) no determinística y (iii) móvil. En el despliegue determinístico, la ubicación de los nodos sensores está determinada desde el diseño de la aplicación, donde los nodos se instalan manualmente y se les asignan rutas estáticas, como, por ejemplo, en aplicaciones industriales, de monitorización de infraestructuras, entre otras aplicaciones de tipo indoor o áreas de extensión reducida. En una implantación no determinística, las redes de sensores inalámbricas tienen la característica de ser desplegadas en ambientes dinámicos (con muchos eventos), remotos, a veces hostiles y de difícil acceso, donde la presencia de un humano para realizar mantenimiento no es una buena práctica. Por esa razón, se necesita que las aplicaciones sean autónomas, evitando al máximo la dependencia de un humano. Para que una red de sensores inalámbrica sea autónoma debe ser autoconfigurable, autocurable, autoptimizable y autoprotegible [17]. Por último, en los despliegues móviles, los nodos sensores pueden moverse activamente para cubrir áreas de interés, o moverse de forma pasiva por la acción de fuerzas externas como animales, deslizamiento de tierra o inundaciones.

Los escenarios de aplicación de las redes de sensores inalámbricas son muy variados [18], se pueden implementar en agricultura [19, 20], soluciones biométricas [21], ciudades inteligentes [22] [23], control de infraestructuras [24-26], domótica [27], entornos inteligentes, industria [28], logística [29], medioambiente [30], en temas militares [31], salud [32, 33], e inclusive seguridad [34, 35].

En lo últimos años, junto al crecimiento de los escenarios citados anteriormente, un nuevo paradigma denominado Internet de las Cosas (IoT, por sus siglas en inglés, Internet of Things), viene tomando relevancia en la industria, la academia y en áreas del saber relacionadas con la tecnología. El ITU-T define al IoT como una infraestructura global que permite ofrecer servicios avanzados mediante la interconexión de objetos físicos y virtuales, gracias a la interoperabilidad de tecnologías de la información 
y la comunicación (TIC) presentes y futuras, que aprovecha las capacidades de identificación, adquisición de datos, procesamiento y comunicación para ofrecer servicios a todos los tipos de aplicaciones garantizando a su vez los requisitos de seguridad y privacidad [36].

Así como ocurre en las aplicaciones para redes de sensores inalámbricas, las aplicaciones de IoT también son muy variadas, entre ellas se encuentran la agricultura [37], ciudades inteligentes [38], control de infraestructuras [39], casas inteligentes [40], entornos inteligentes, industria [41], logística [42], medioambiente [43], soluciones militares [44], en el campo de la salud [45], y seguridad [46].

Como se puede observar, las redes de sensores inalámbricas e Internet de las Cosas comparten los mismos escenarios de aplicación citados y buscan proponer soluciones a problemas con las mismas características y, si se hace una revisión de las soluciones propuestas bajo el paradigma de Internet de las Cosas por la industria, la academia o la vinculación de estas dos, se puede observar que muchas de estas soluciones integran redes de sensores inalámbricas.

Lo anterior da pie a que se suscite una ambigüedad entre los conceptos, y precisamente el tema de discusión de este artículo es buscar responder estas preguntas: ¿Internet de las Cosas y las redes de sensores inalámbricas son términos equivalentes?, ¿Internet de las Cosas engloba a las redes de sensores inalámbricas?, o si estas son complementarias o rivalizan entre sí. Este trabajo tiene como objetivo presentar, analizar y comparar las características propias de WSN e IoT para ofrecer un panorama general de sus similitudes y diferencias en torno a cinco temas, a saber: (i) nivel conceptual, (ii) requisitos generales, (iii) arquitecturas, (iv) construcción de aplicaciones y (v) tratamiento de los datos.

El resto del artículo está organizado en siete secciones directamente relacionadas con los temas analizados, a saber: definiciones y tendencias, arquitecturas, requisitos generales de aplicación, enfoques de construcción y enfoques hacia los datos tanto en WSN como en IoT. Finalmente, se da lugar una discusión y a las conclusiones derivadas de la reflexión.

\section{Definiciones y tendencias}

Según una recomendación de la ITU-T [6] una red de sensores inalámbrica es una red compuesta de nodos sensores interconectados que intercambian datos detectados por vía inalámbrica. Para Yick et al. [47] la WSN consiste en un número de nodos sensores que puede variar de unas pocas decenas hasta miles, que trabajan juntos para supervisar una región con el fin de obtener datos sobre el medioambiente. Además, los autores definen dos tipos de redes inalámbricas de sensores: estructuradas y no estructuradas. Una WSN no estructurada contiene una densa colección de nodos de sensores que pueden ser desplegados de manera ad-hoc en el campo, y la cual se deja desatendida para realizar funciones de supervisión y generación de informes, lo cual dificulta el mantenimiento de la red. En una WSN estructurado, todos o algunos de los nodos del sensor se despliegan de una manera preplanificada, lo que significa la implementación de un menor número de nodos, un menor coste de mantenimiento y gestión de la red y se puede proporcionar una mejor cobertura.

En cuanto a IoT, no existe una definición estandarizada, por lo que varias instituciones han definido un acercamiento conceptual de su visión de este paradigma. Para Gartner Inc. [48] y GSMA [49], Internet de las Cosas es una red de dispositivos físicos conectados de forma inteligente con la capacidad de recolectar datos del ambiente; Además, para Gartner, IoT es un ambiente en el cual interactúan las cosas, las comunicaciones, y las aplicaciones, y se realiza un análisis de datos recolectados. Otras instituciones como CERP-IoT [50] y Casagras [51], tienen un concepto mucho más amplio, y definen a IoT como una infraestructura de red global, dinámica y autonómica, en el cual, interactúan cosas físicas y virtuales que tienen una identidad propia, con un alto grado de autonomía de captura de datos, transferencia de eventos, conectividad de red e interoperabilidad.

Analizando estos conceptos dados sobre WSN e IoT, los nodos sensores dentro de una red WSN pueden monitorizar e interactuar entre ellos al igual como lo hacen los objetos físicos y virtuales en IoT, pero, para que estos objetos puedan interactuar entre sí, se hace necesario que los objetos integren un nodo sensor que les dé la capacidad de monitorizar, procesar y comunicar los datos obtenidos. Una vez se cumpla con este requisito, una aplicación IoT podrá monitorizar (o sentir) las condiciones físicas o 
ambientales de la zona geográfica en que se encuentra al igual que una aplicación WSN. La síntesis de estos dos conceptos se muestra en la Tabla 1.

Tabla 1. Análisis de características de WSN e IoT. Fuente: Autores.

\begin{tabular}{l|l|lll}
\hline \multicolumn{1}{c|}{ Característica } & \multicolumn{1}{|c|}{ WSN } & \multicolumn{2}{c}{ IoT } & \\
\hline Definición estandarizada & Sí & No & \\
\hline Infraestructura de red & Local & Global & \\
\hline Red autónoma & Sí & Sí & \\
\hline Objetos & Físicos & Físicos y virtuales & \\
\hline Análisis de los datos & No & Sí & & \\
\hline Identidad de los objetos & Sin & $\begin{array}{l}\text { Identidad propia dentro de } \\
\text { Internet, cada objeto es } \\
\text { identificado }\end{array}$ \\
\hline
\end{tabular}

Para analizar las tendencias de estas dos áreas de estudio se revisó el número de publicaciones científicas realizadas entre los años 1980 y 2017, y se tuvieron en cuenta todos los tipos de documentos generados en el periodo consultado. Para ello, se usó la base de datos especializada Scopus, la cual permite analizar los resultados obtenidos para cada una de estas áreas y generar la gráfica que se muestra en la Figura 1.

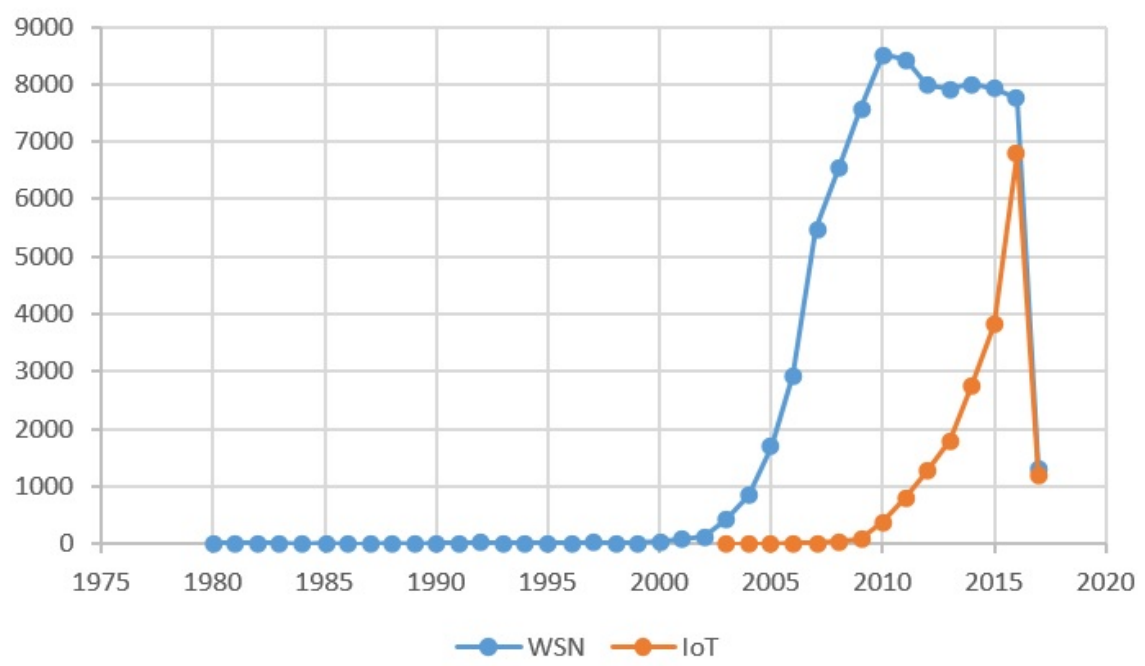

Figura 1. Desarrollo y tendencia de WSN e IoT. Fuente: Scopus.

En color azul traza la tendencia que ha tenido WSN hasta la actualidad, y se puede observar que hasta el año 2001 las WSN no representaban un tema relevante en el entorno académico, con solo 74 publicaciones. Pero a partir del año 2002 su popularidad fue aumentando hasta llegar a su pico máximo en el año 2010 con 8.514 publicaciones y, a partir de allí, ha venido descendiendo paulatinamente, y se han registrado 7.758 publicaciones en el año 2016, y en el primer trimestre del 2017 alrededor de 1.300 publicaciones.

La tendencia de IoT, en color naranja, comienza en 2003. En este año se registraron 3 publicaciones y, aunque en 2005 se conoció la primera definición formal sobre IoT por parte de la ITU-T, su popularidad comenzó a crecer hacia el año 2010, en el cual se registraron 390 publicaciones y ha venido creciendo exponencialmente hasta alcanzar 6.786 en el año 2016. En el primer trimestre del 2017 se registran alrededor de 1.170 publicaciones. 
Analizando estas tendencias, se puede afirmar que el aumento de la popularidad de IoT no ha afectado el desarrollo de la investigación de WSN y, aunque las aplicaciones IoT hacen uso de nodos sensores y sus redes para que los objetos puedan interactuar con el medio y comunicarse con otros objetos, las WSN como área de estudio no ha perdido su relevancia en la industria y la academia. El pequeño descenso que han tenido publicaciones en WSN puede ser explicado por esa integración que se menciona anteriormente; las aplicaciones de WSN han comenzado a verse como una parte del paradigma de IoT y las nuevas soluciones se van orientando hacia ese paradigma, en el cual se toman las ventajas ofrecidas por WSN y se integran con otras ventajas que ofrecen otro tipo de tecnologías y que se integran como un todo en IoT.

\section{Requisitos generales de aplicación}

Los requisitos generales hacen referencia a los requisitos que toda aplicación debe cumplir, sin importar el escenario en que se implemente. Para Hadim y Nohamed [52, 53], entre los requisitos que debe cumplir una aplicación de redes de sensores inalámbricas están: (i) uso eficiente de energía y recursos limitados, fundamental para garantizar la vida útil de la solución, ya que en muchos casos estas se implementan en áreas remotas, sin intervención del ser humano salvo para su implantación; (ii) escalabilidad, donde la red debe ser lo suficientemente flexible como para permitir el crecimiento en cualquier lugar y momento sin afectar su rendimiento; (iii) movilidad, (iv) topologías dinámicas para la red, (v) heterogeneidad, (vi) organización en red, (vii) la agregación de datos, (viii) la calidad del servicio, (ix) la seguridad, (x) la integración con el mundo real.

Para Borgia [54], una aplicación basada en el paradigma de IoT debe cumplir unos requisitos generales, tales como: (i) heterogeneidad, para la gestión de la variedad de dispositivos, tecnologías, servicios y ambientes; (ii) escalabilidad; (iii) minimización de costos de desarrollo, mantenimiento y consumo de energía; (iv) autonomía, es decir, la aplicación debe ser autoconfigurable, autorganizable, autoadaptable, con capacidad de autoreacción a los acontecimientos y estímulos, con capacidad de autodescubirmiento de entidades y servicios, y (v) tener la capacidad para el procesamiento de grandes volúmenes de datos.

Como se puede observar, los requisitos de aplicación entre las WSN e IoT son muy similares, la diferencia radica en el grado de relevancia que tiene en cada una de ellas. En la Tabla 2 se presentan los requisitos de aplicación comunes a las WSN e IoT con el respectivo nivel de relevancia que el requisito tiene en cada una de ellas. La relevancia de cada requisito se representa en cuatro niveles: alta, media, baja y nula.

Tabla 2. Requisitos de aplicación en WSN e IoT. Fuente: Autores.

\begin{tabular}{l|l|l}
\hline Requisitos & WSN & IoT \\
\hline Gestión de recursos & Media & Alta \\
\hline Gestión de costos & Media & Alta \\
\hline Autonomía & Alta & Alta \\
\hline Escalabilidad & Alta & Alta \\
\hline Movilidad & Media & Media \\
\hline Topologías de red dinámica & Alta & Baja \\
\hline Heterogeneidad & Media & Alta \\
\hline Organización de red & Alta & Media \\
\hline Agregación de datos & Alta & Media \\
\hline Calidad del servicio & Alta & Alta \\
\hline Seguridad & Media & Alta \\
\hline $\begin{array}{l}\text { Autoprocesamiento de grandes volúmenes de } \\
\text { datos }\end{array}$ & Nula & Alta \\
\hline
\end{tabular}

Los requisitos de gestión de recursos, gestión de costos y heterogeneidad son altos en IoT porque su infraestructura es mucho más extensa, su ecosistema es más variado en dispositivos, tecnologías y 
servicios. En ambas áreas de estudio, la autonomía, la escalabilidad y la calidad del servicio tienen una alta relevancia en sus aplicaciones.

\section{Arquitecturas}

Las aplicaciones de WSN e IoT, como todo sistema, tienen una arquitectura que define cómo es la estructura lógica y física de los componentes que la integran y cómo estas interactúan entre sí para lograr el objetivo de dicho sistema.

La International Telecommunication Union (ITU-T) define que la arquitectura de una aplicación IoT generalmente está compuesta de cuatro capas: Capa de aplicación, capa de apoyo a servicios y aplicaciones, capa de red y capa de dispositivo; y dos módulos trasversales: el de gestión y el de seguridad [36], como se muestra en la Figura 2. Pero esta no es la única arquitectura propuesta para IoT. Otros investigadores y organizaciones han propuesto sus arquitecturas entre la que encontramos las propuestas por la European FP7 Research [50], la de la IoT World Forum [55], la de la Industrial Internet Consortium [56], por solo nombrar algunas de ellas.

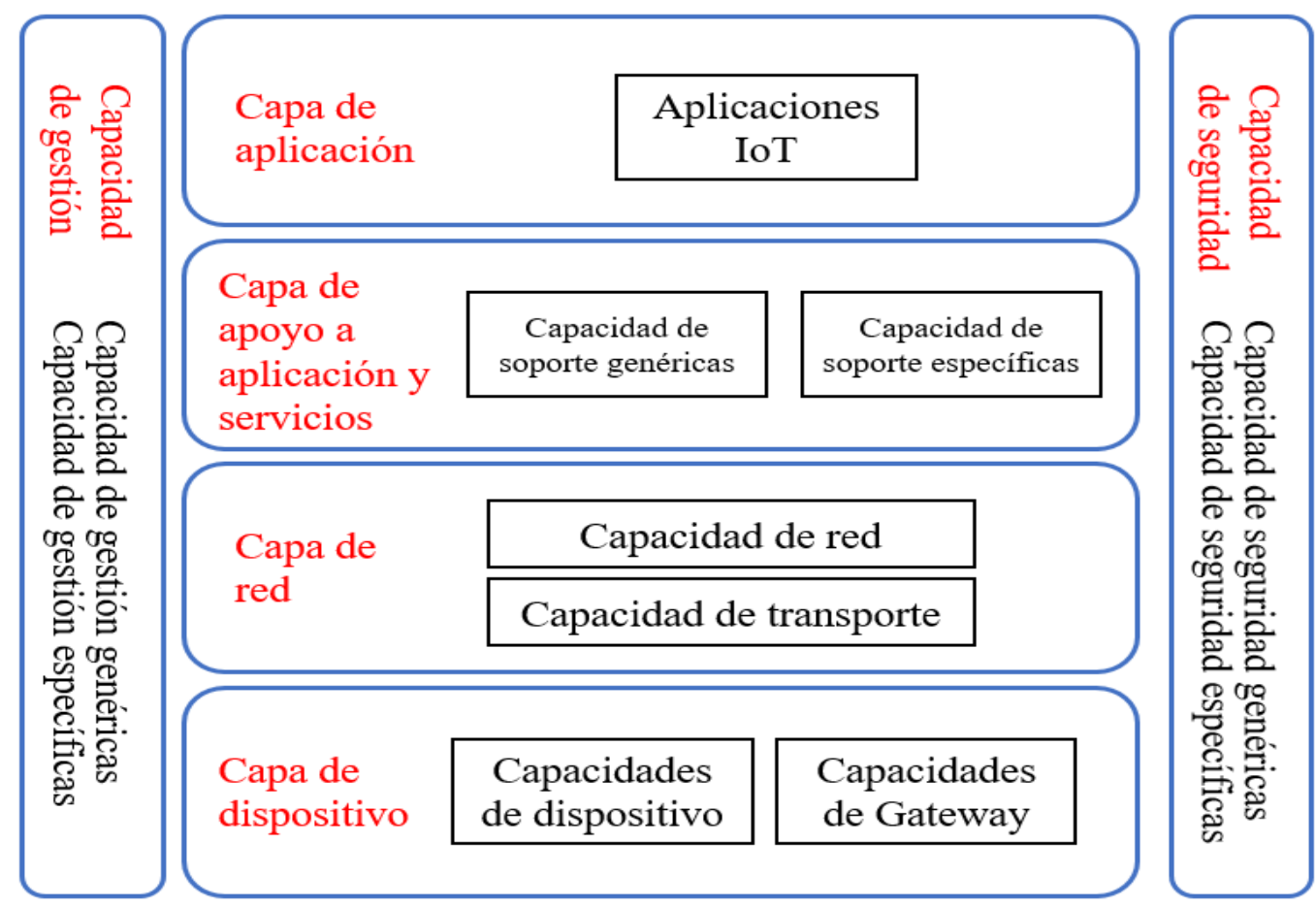

Figura. 2. Modelo de referencia de IoT. Fuente: ITU-T [36].

En cuando a las WSN, estas no tienen una arquitectura compleja, y según Yick et al. [47], estas redes normalmente tienen poca o ninguna infraestructura. Su arquitectura hace énfasis en la capa de dispositivos o física y es allí donde se concentra la mayor parte de una aplicación de redes de sensores inalámbricas, como se muestra en la Figura 3. 

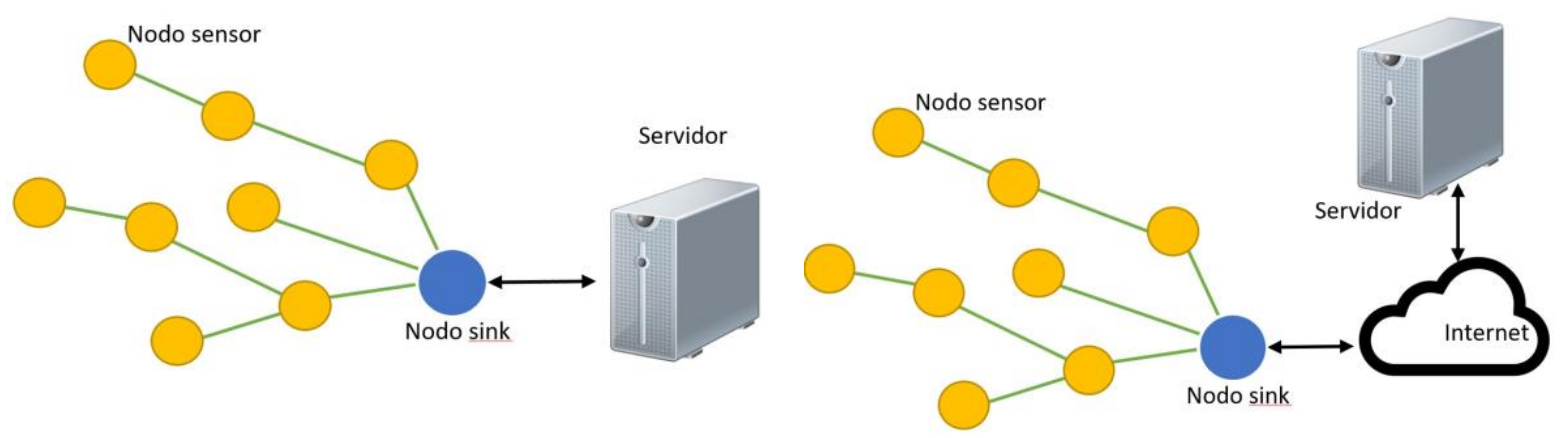

Figura 3. (a) Arquitectura básica de WSN. (b) Fuente Arquitectura WSN usando Internet Adaptado de [18].

Existen varios tipos de estructuras para organizar una WSN [18], la más común es la que se muestra en la Figura 3(a). Las aplicaciones WSN cuentan con una de nodos sensores y nodo sink. El nodo sink, es un nodo con mayores capacidades, el cual es el encargado de recibir los datos enviados por los nodos y enviarlos al servidor en el cual se podrán consultar y visualizar los datos. También existen otras dos posibilidades para una arquitectura WSN: la primera es que se use un gateway de campo que comunique la red de nodos sensores con el servidor, de este modo, los nodos sink envían sus datos al gateway y este envíe los datos al servidor; la segunda es usar la red Internet para enviar estos datos al servidor, como se muestra en la Figura 3(b). La nube tiene gran relevancia en aplicaciones de IoT, pues este componente soporta una gran infraestructura, con componentes de almacenamiento y analítica, entre otros. Mientras que, comúnmente, la nube en una aplicación de redes de sensores inalámbricos apenas es el canal de comunicación entre la red de sensores y el usuario y su función es permitir la visualización de los datos.

Respecto de las arquitecturas, ya sean para WSN e IoT, se habla de dos tipos de requisitos: los generales y específicos. Los requisitos generales hacen referencia a los requisitos que toda aplicación de WSN e IoT debe cumplir, sin importar el escenario en que se implemente. Los requerimientos específicos son aquellos requisitos que tienen una aplicación en un escenario particular, por ejemplo, una aplicación para la seguridad física o perimetral no tiene los mismos requerimientos específicos que una aplicación para el área de salud, vestibles o agricultura.

Para el análisis y comparación de las arquitecturas de WSN e IoT se tomó como referencia las cuatro capas de la arquitectura presentada por la ITU-T [36]: capa de aplicación, capa de apoyo a servicios y aplicaciones, capa de red y la capa de dispositivos. A continuación, se presenta una breve descripción de cada una de estas capas:

- Capa de Aplicación. En esta capa se encuentran los componentes propios de cada implementación que permiten interactuar con el resto de la aplicación, tales como los componentes de gestión, configuración y seguridad, más los componentes de visualización de datos. Los escenarios de aplicación son muy variados: agricultura y medioambiente, ciudades inteligentes, logística, salud, seguridad y vestibles. Pero dependiendo del escenario, puede predominar las WSN o IoT, el cual, además de estas redes integra otro tipo de tecnologías como la RFID, sistemas de middleware, la computación en la nube y las aplicaciones [58].

- Capa de apoyo a servicios y aplicaciones. En esta capa se tienen en cuenta la infraestructura, la localización de dicha infraestructura dentro de la arquitectura y la relevancia que tiene el middleware.

- Capa de red. En esta capa se realiza la agregación de datos y se encuentran las tecnologías de comunicación, los algoritmos de agregación, enrutamiento y diseminación, de control de topologías y los protocolos y formatos para el intercambio de mensajes.

- Capa de dispositivos. En esta capa se encuentran los dispositivos físicos que soportan la aplicación, como son los nodos sensores, y se gestiona la identificación de los mismos, las capacidades de hardware que estos tienen y el recurso de energía. 
En cada una de estas capas se presentan características comunes o diferenciadoras para estas dos áreas de estudio. En la tabla 3 se realiza un análisis donde se determinan sus diferencias, enfoques y tecnologías usadas en cada uno de ellos, facilitando la identificación de las diferencias entre la arquitectura de una aplicación de red de sensores inalámbrica y la arquitectura de una aplicación en Internet.

\section{Enfoque de construcción}

Para la construcción de una solución se encuentran dos enfoques: uno de abajo hacia arriba o Buttom-Up y dos, el de arriba hacia abajo o Top-Down. El enfoque Buttom-Up se aplica a las WSN, esto se debe a las limitaciones de recursos que tienen los nodos sensores. Sus requerimientos principales se centran en resolver los problemas de la capa de dispositivos. De esta forma, para diseñar una aplicación primero se deben resolver estos problemas en la capa más baja e ir subiendo a las diferentes capas hasta llegar a la de aplicación, hasta lograr que esta soporte todos los requisitos de hardware de los nodos sensores.

Tabla 3. Comparación de las arquitecturas de WSN e IoT, a nivel de enfoque y tecnologías usadas. Fuente: Autores.

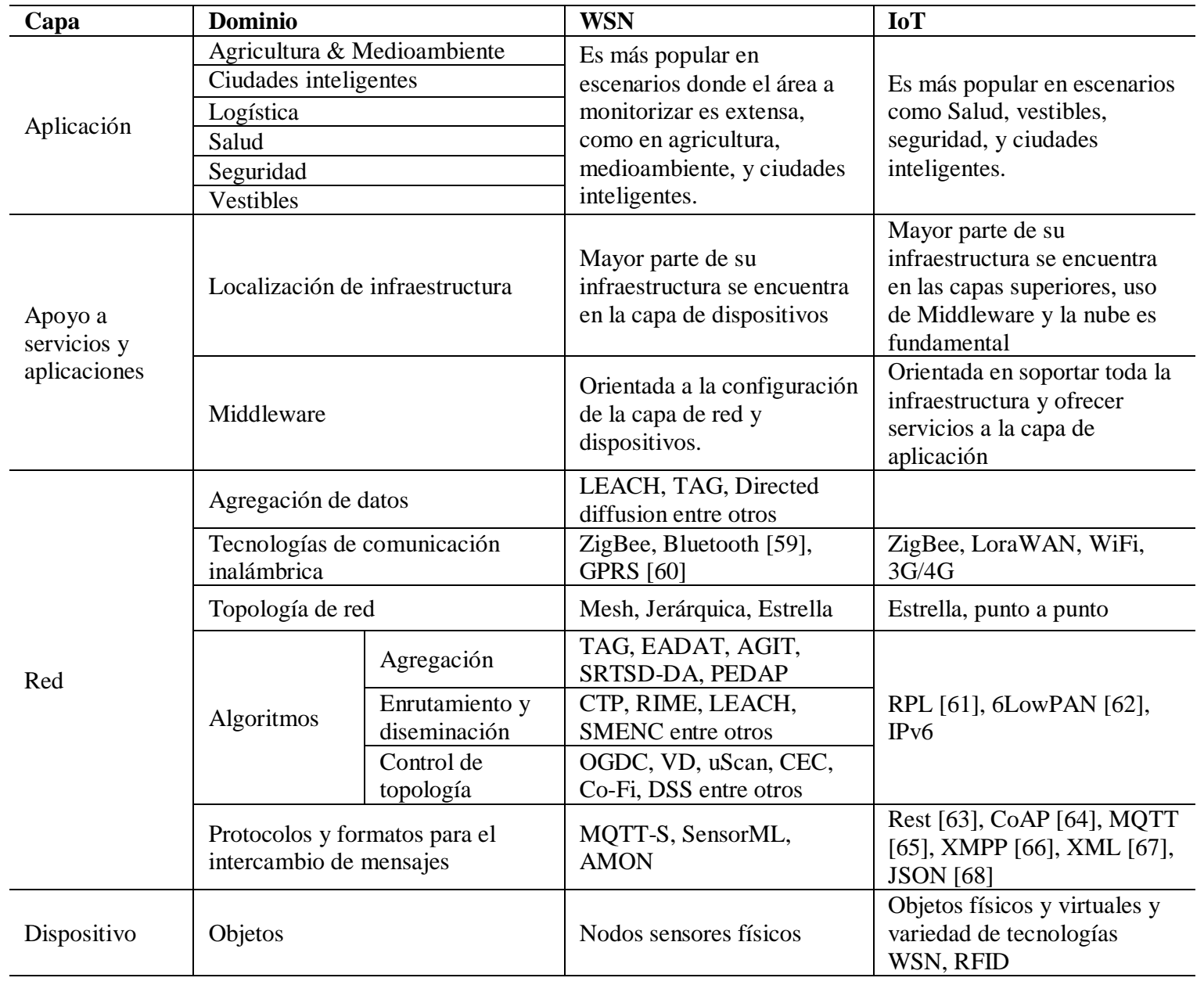




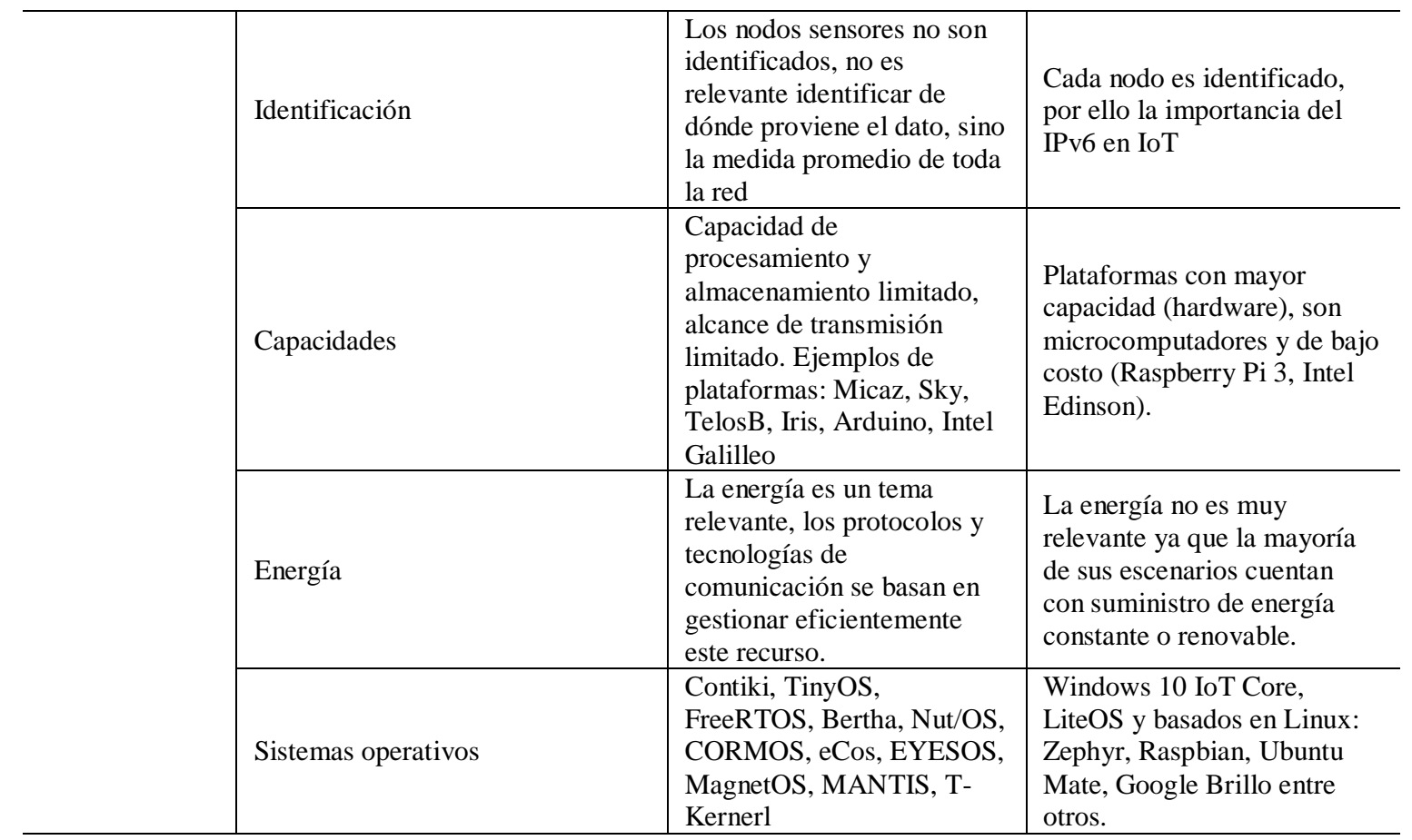

Por otro lado, el enfoque Top-Down se aplica a la construcción de aplicaciones IoT, en el cual no existe la misma preocupación sobre la limitación de los recursos de hardware y las aplicaciones son construidas teniendo en cuenta los requerimientos de la solución. Posteriormente, se construye la arquitectura y con base en los factores anteriores se definen las características de los dispositivos que estarán en la capa de dispositivos y la tecnología que estará en cada uno de los niveles de la arquitectura.

Los dos enfoques anteriores definen la forma en que son construidas las aplicaciones, teniendo en cuenta los requerimientos de cada uno de estas áreas de estudio. Pero existen otros dos enfoques que tratan de la forma como se dispone la infraestructura de las tecnologías de información y la comunicación (TIC). Borgia [54] refiere estos dos enfoques como enfoque vertical y enfoque horizontal. En el enfoque vertical, cada aplicación es construida con su propia infraestructura TIC y dispositivos dedicados, como se muestra en la Figura 4, y las aplicaciones similares no comparten ninguna característica para la gestión de servicios y la red, lo que resulta en la redundancia innecesaria y aumento de los costos. En el enfoque horizontal, una plataforma operativa común gestiona la red y los servicios y abstrae los datos de una amplia gama de fuentes para permitir el correcto funcionamiento de las aplicaciones, ver Figura 5.

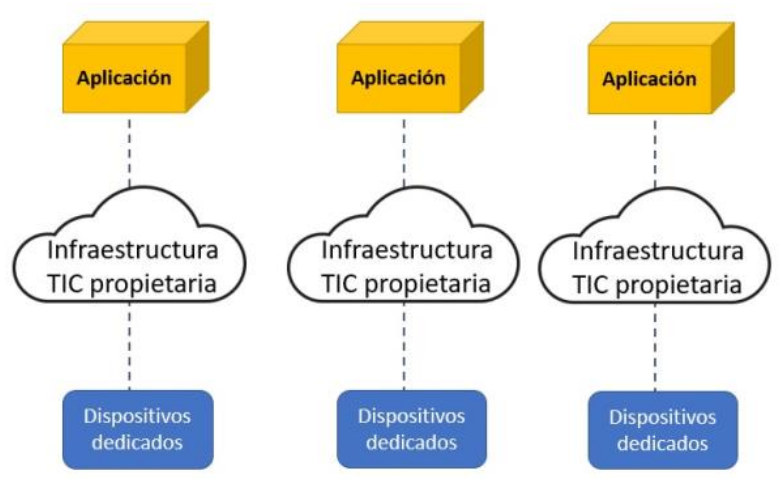


Figura 4. Enfoque vertical. Fuente: Adaptado de Biorgia [54].

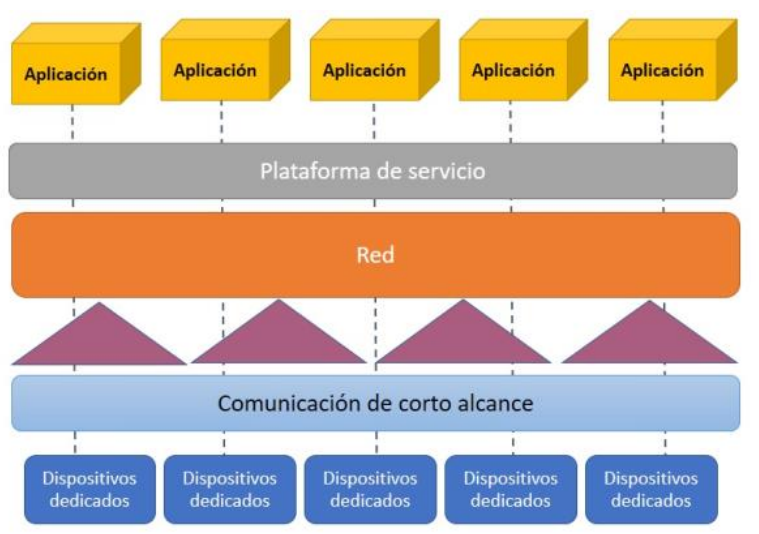

Figura 5. Enfoque horizontal. Fuente: Adaptado de Biorgia [54].

En el enfoque vertical para WSN, cada aplicación gestiona de forma independiente toda la complejidad de comunicación y gestión de recursos limitado, es decir, en cada solución se desarrolla la plataforma para la gestión de la red. Para facilitar la gestión de las comunicaciones y los recursos de la red, en WSN también se ha desarrollado el enfoque horizontal a través de las aplicaciones middleware. Un middleware es un software que proporciona una plataforma común para la heterogeneidad, permitiendo que múltiples aplicaciones funcionen en paralelo en una red distribuida, e intenta resolver los problemas de diseño e implementación de las aplicaciones WSN [69]. Se ha propuesto una variedad de middlewares para WSN [70], cuyo propósito en facilitar la construcción de una solución WSN al brindar a los desarrolladores la posibilidad de crear aplicaciones sin tener que preocuparse por las capas inferiores. Estos middlewares ya tienen en cuenta los requerimientos de gestión de la red, en cuanto a la comunicación entre los nodos, la solicitud de datos de los nodos y el uso eficiente de los recursos.

En IoT, el enfoque horizontal y el vertical tienen algo en común, ambos hacen uso de la computación en la nube, el cual es un requisito en este paradigma. La diferencia se encuentra en la implementación de la infraestructura tecnológica que, en el enfoque vertical, cada aplicación tiene su propia infraestructura, mientras que, en el horizontal, se comparte la infraestructura para la gestión de la red y otros servicios que soportan una variedad de aplicaciones y están orientados a ofrecer servicios complejos desde el lado de nube. En la Tabla 4 se sintetizan las diferencias entre los enfoques vertical y horizontal en WSN e IoT.

Tabla 4. Comparación de los enfoques vertical y horizontal en WSN e IoT. Fuente: Autores.

\begin{tabular}{|c|c|c|c|c|c|}
\hline \multirow[b]{2}{*}{ Característica } & & \multicolumn{2}{|l|}{ WSN } & \multicolumn{2}{|l|}{ IoT } \\
\hline & & Enfoque vertical & Enfoque horizontal & Enfoque vertical & $\begin{array}{l}\text { Enfoque } \\
\text { horizontal }\end{array}$ \\
\hline Alcance & & Local & Local & Global & Global \\
\hline Plataforma & & $\begin{array}{l}\text { Específica para la } \\
\text { gestión de la red }\end{array}$ & $\begin{array}{l}\text { Uso de middleware, } \\
\text { pero con alcance } \\
\text { local }\end{array}$ & $\begin{array}{l}\text { Propia basada en la } \\
\text { cloud }\end{array}$ & $\begin{array}{l}\text { Común a varias } \\
\text { aplicaciones, } \\
\text { basada en la cloud }\end{array}$ \\
\hline Servicios & & $\begin{array}{l}\text { Gestión de la comu } \\
\text { limitados }\end{array}$ & icación y recursos & $\begin{array}{l}\text { Servicios más co } \\
\text { administración y a } \\
\text { volúmenes de datos }\end{array}$ & $\begin{array}{l}\text { nplejos como la } \\
\text { alítica de grandes }\end{array}$ \\
\hline $\begin{array}{l}\text { Ventajas } \\
\text { limitaciones }\end{array}$ & $\mathrm{y}$ & 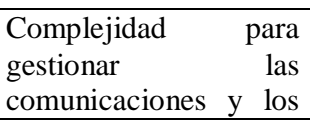 & $\begin{array}{lr}\text { Facilidad } & \text { para } \\
\text { gestionar } & \text { las } \\
\text { comunicaciones } & y\end{array}$ & $\begin{array}{lr}\text { Redundancia } & \text { de } \\
\text { infraestructura } & y \\
\text { servicios } & y\end{array}$ & $\begin{array}{l}\text { Reducción de los } \\
\text { costos al compartir } \\
\text { infraestructura y }\end{array}$ \\
\hline
\end{tabular}




\begin{tabular}{l|l|l|l|l|l}
\hline & recursos & $\begin{array}{l}\text { los recursos, } \\
\text { reduciendo tiempo y } \\
\text { costos }\end{array}$ & $\begin{array}{l}\text { aumento de los } \\
\text { costos }\end{array}$ & $\begin{array}{l}\text { servicios y su } \\
\text { respectiva } \\
\text { administración }\end{array}$ \\
\hline
\end{tabular}

\section{Enfoque hacia los datos}

Sobre la importancia que estos dos campos de estudio han dado a los datos, se identifica que WSN está centrada en los datos. Es decir, a sus aplicaciones solo les interesa la recolección coordinada de los datos dentro del área geográfica y no de qué nodo sensor provienen esos datos. Para realizar la recolección coordinada de datos la WSN aplica diferentes técnicas de fusión y agregación de datos [71] [72] [73] con el propósito de dar un único valor representativo del área en cuestión. Por otro lado, para una aplicación IoT, los datos tienen un enfoque más relevante que una simple medida de una variable, también le es relevante conocer de qué nodo sensor proviene (esta es la razón de que cada objeto tiene una identificación), y de esta forma, un nodo sensor al detectar un evento crea un mensaje con el valor obtenido de la condición física que está monitorizando y la dirección física del propio sensor.

Por ello, para IoT es de suma importancia el protocolo de Internet versión 6 (IPv6) [74], porque soporta un número mayor de nodos direccionales al incrementar el tamaño de la dirección IP actual (IPv4) de 32 bits a 128 bits y simplifica la autoconfiguración de direcciones. De esta forma se garantiza que cada objeto tenga una dirección que sea identificable con Internet.

Otro punto diferenciador entre las WSN e IoT es el tratamiento que le hacen a los datos obtenidos por las capas de dispositivos de sus respectivas aplicaciones. Mientras que en una aplicación WSN se monitorizan las condiciones físicas, se recolectan los datos y se comunican con la aplicación, en el paradigma de IoT los datos por sí solos no son tan valiosos como para una WSN. Para una aplicación IoT, aparte de tener una medida, el verdadero valor de los datos radica en el procesamiento y análisis que se pueda realizar a estos, usando técnicas de big data, pasando el dato a la información, luego al conocimiento y llevarlo a un nivel más superior, a la sabiduría [75]. Las figuras 6 y 7 muestran de forma gráfica el enfoque que tienen los datos en las WSN e IoT.

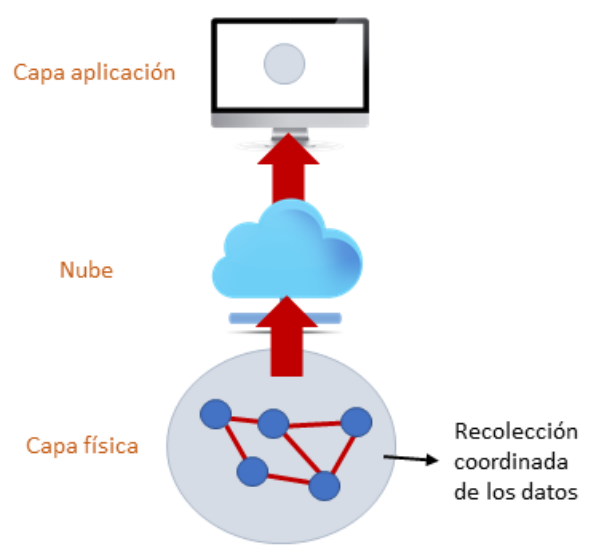

Redes de sensores inalámbricas

Figura 6. Adquisición de datos en WSN. Fuente: Autores. 


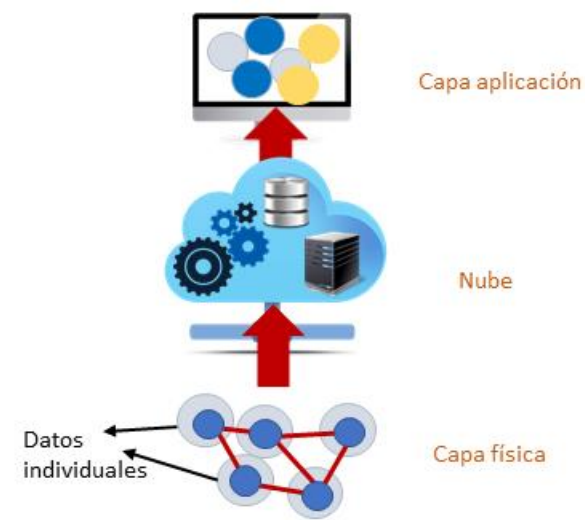

Internet de las Cosas

Figura 7. Adquisición de datos en IoT. Fuente: Autores.

En la Figura 6 se puede observar la adquisición de datos en las WSN. La red de nodos sensores recolecta los datos de forma coordinada y son enviados a través de la nube para su organización y visualización desde la capa de aplicación. La nube funciona como un canal de comunicación para permitir a los administradores acceder a los datos de forma remota.

En la Figura 7 se muestra cómo es la adquisición de datos en IoT. En este paradigma, los datos son adquiridos por el dispositivo y es identificado cada uno de ellos. Estos dispositivos envían un paquete con el valor del dato obtenido más su identificación única. Todos esos datos son enviados hacia la nube, ya sea directamente desde el dispositivo o a través de un gateway que recibe los datos de los dispositivos, los agrupa y los envía a la nube. La nube en IoT, a diferencia de WSN, es mucha más que un canal de comunicación y soporta toda una infraestructura tal como es la red, el procesamiento y el almacenamiento de los datos y su posterior análisis usando técnicas de big data. A través del big data los datos recolectados se analizan generando nuevos datos y es allí donde radica la relevancia de este paradigma. La capa de aplicación organiza toda esa información para facilitar su comprensión por parte de los usuarios y ayudar en la toma de decisiones.

\section{Discusión}

De acuerdo a [76], las WSN e IoT tienen un origen en común: las redes de computadoras y la comunicación máquina-a-máquina o M2M (por sus siglas en inglés Machine-to-Machine). Esto se refleja en que estos dos campos de estudio compartan características, requerimientos y dominios de aplicación, pero diferenciándose en los enfoques que cada uno de ellos tiene.

En este orden de ideas, se puede afirmar que las WSN fueron integradas por el paradigma del IoT, pasando a ser parte de las comunicaciones en el ecosistema de tecnologías que este paradigma integra. En los trabajos realizados por I. Lee y K. Lee [58], y Pandya y Champaneria [77], presentan a las redes de sensores inalámbricas como una tecnología fundamental del IoT, junto a otras tecnologías como RFID, sistemas de middleware, computación en la nube y las aplicaciones.

Para la IEEE existe una clara diferencia entre las WSN e IoT: el alcance de las WSN es la recolección coordinada de los datos, mientras que, en IoT, la identificación única de las cosas y su conexión a Internet es una característica necesaria para que Internet de las Cosas no esté relacionada con las WSN [78]. Otro factor diferenciador entre WSN e IoT es la forma como son diseñadas y construidas las aplicaciones, cada uno le da un enfoque diferente. 


\section{Conclusiones}

Las WSN e IoT tienen su origen en las redes de computadoras y la comunicación máquina-a-máquina, y, aunque comparten características, requerimientos y dominios de aplicación, estos campos de estudio no con equivalentes en todos los aspectos, sino que cada uno de ellos ha evolucionado tomando enfoques diferentes. Teniendo en cuenta estos enfoques se puede concluir lo siguiente:

Primero, IoT no es una nueva tecnología en sí misma, sino que es un nuevo paradigma que integra tecnologías ya existentes y que posiblemente integre otras tecnologías que actualmente están en desarrollo o serán desarrolladas en los próximos años. Por esta razón, WSN e IoT no rivalizan entre sí, sino que WSN enriquece el ecosistema IoT, aportando sus funcionalidades, sus ventajas y la capacidad de integrarse dentro de una misma aplicación con otro tipo de tecnologías.

Segundo, WSN e IoT comparten los requerimientos de aplicación, pero dentro de cada campo la relevancia de estos requerimientos puede variar, dependiendo de sus necesidades y limitaciones. Estos requerimientos determinan la forma en que se construye una aplicación WSN o una aplicación IoT y se deben tener en cuenta desde la fase de diseño de la aplicación.

Tercero, que WSN e IoT no son términos equivalentes por más similitud que se pueda percibir a primera vista. Estas dos áreas de estudio tampoco están contendiendo entre sí, sino que, dependiendo del dominio de aplicación, uno de estos campos puede ser más implementado que el otro, pero con la tendencia creciente que ha tenido IoT en los últimos años las aplicaciones en las cuales convergen estos campos de estudio son cada vez más comunes y en una gran variedad de dominios de aplicación.

Cuarto, que a pesar de que el paradigma de IoT cobijó a las WSN, no se puede pensar que las WSN dejarán de ser investigadas como un área de estudio por sí misma y pasarán a ser una tecnología obsoleta, sino que, como un campo de estudio independiente, sigue siendo un tema de constante investigación y desarrollo, trabajando en diferentes áreas como son el desarrollo de nodos sensores más potentes y eficientes y redes más autónomas. Esto sin duda también beneficiará el desarrollo del paradigma de IoT.

En resumen, el Internet de las cosas integra a las redes de sensores inalámbricos de la misma forma que integra otras tecnologías como es el caso de los sistemas RFID, los sistemas de middleware y la Computación en la Nube y futuras tecnologías y conceptos tecnológicos que puedan surgir y el desarrollo de cada una de estas tecnologías ayudará a la evolución del Internet de las Cosas, complementando paralelamente el crecimiento de las demás tecnologías que integren el paradigma que actualmente reina en el ámbito académico e industrial.

\section{Agradecimientos}

Los autores desean agradecer la colaboración de todos los socios dentro del proyecto Centro de Excelencia y Apropiación en Internet de las Cosas (CEA-IoT). Los autores también desean agradecer a todas las instituciones que apoyaron este trabajo: el Ministerio de Tecnología de la Información y Comunicaciones - MinTIC de Colombia y al Departamento Administrativo de Ciencia, Tecnología e Innovación - Colciencias, a través del Fondo Nacional de Financiamiento para la Ciencia, Tecnología y la Innovación Francisco José de Caldas (ID Proyecto: FP44842-502-2015).

\section{Referencias}

[1] R. Wesson, F. Hayes-Roth, J. W. Burge, C. Stass y C. A. Sunshine, «Network Structures for Distributed Situation Assessment,» IEEE Transactions on Systems, Man, and Cybernetics, vol. 11, $\mathrm{n}^{\circ}$ 1, pp. 5 - 23, 1981.

[2] C.-Y. Chong, K.-C. Chang y S. Mori, «Distributed Tracking in Distributed Sensor Networks,» de American Control Conference, 1986, Seattle, WA, USA, 1986.

[3] C.-Y. Chong, S. Mori y K. Chang, «Information fusion in distributed sensor networks,» de American Control Conference, Boston, MA, 1985. 
[4] C. Chong, S. Mori, E. Tse y R. Wishner, «Distributed Estimation in Distributed Sensor Networks,» de American Control Conference, 1982, Arlington, VA, USA, 1982.

[5] International Telecommunication Union, «Serie Y.2121: Requirements for support of ubiquitous sensor network (USN) applications and services in the NGN environment,» Telecomunication Standardization Sector of ITU, 2010.

[6] International Telecommunication Union, «Requirements for support of ubiquitous sensor network (USN) applications and services in the NGN environment (Y.2221),» Telecommunication Standardization Sector of ITU , 2010.

[7] I. Akyildiz, W. Su, Y. Sankarasubramaniam y E. Cayirci, «Wireless sensor networks: a survey,» Computer Networks, vol. 38, pp. 393-422, 2002.

[8] X. Chen, X. Wang y X. Chen, «Energy-Efficient Optimization for Wireless Information and Power Transfer in Large-Scale MIMO Systems Employing Energy Beamforming,» IEEE Wireless Communications Letters, vol. 2, $\mathrm{n}^{\circ}$ 6, pp. $667-670,2013$.

[9] M. Magno , D. Boyle, D. Brunelli y E. Popovici, «Ensuring Survivability of Resource-Intensive Sensor Networks Through Ultra-Low Power Overlays,» IEEE Transactions on Industrial Informatics, vol. 10, n 2, pp. 946 - 956 , 2014.

[10] H. Zainol Abidin, N. M. Din y Y. E. Jalil, «Multi-objective Optimization (MOO) approach for sensor node placement in WSN,» de 7th International Conference on Signal Processing and Communication Systems (ICSPCS), Carrara, VIC, 2013.

[11] G. Jayamurugan y P. Kamalakkannan, «Position-based Key Sharing with Higher Connectivity and Multivariate Optimized Resource Consumption in WSN,» Indian Journal fo Science \& Technology, vol. 8, $\mathrm{n}^{\circ}$ 35, pp. 1-9, 2015.

[12] «Restoring connectivity in a resource constrained WSN,» Journal of Network and Computer Applications, p. $151-165,2016$.

[13] LoRa Alliance, «A technical overview of LoRa ${ }^{\circledR}$ and LoRaWAN" ${ }^{\mathrm{TM}}$,» Technical Marketing Workgroup 1.0, 2015.

[14] ZigBee Alliance, «The ZigBee Alliance,» [En línea]. Available: http://www.zigbee.org/.

[15] IEEE, «IEEE 802.15.4 Part 15.4: Wireless Medium Access Part 15.4: Wireless Medium Access Specifications for Low-Rate Wireless Personal Area Networks (LR-WPANs),» IEEE Computer Society, New York, USA, 2003.

[16] S. Kumar Singh , M. P. Singh y D. Singh, «Routing Protocols in Wireless Sensor Networks - A Survey,» International Journal of Computer Science \& Engineering Survey (IJCSES), vol. 1, nº 2, pp. 63-83, 2010.

[17] J. M. Portocarrero, F. C. Delicato, P. P. F., N. Gámez, L. Fuentes, D. Ludovino y P. Ferreira, «Autonomic Wireless Sensor Networks: A Systematic Literature Review,» Journal of Sensors, vol. 2014, pp. 1-13, 2014.

[18] International Telecommunication Union, «Applications of Wireless Sensor Networks in Next Generation Networks,» Telecommucation Standardization Sector, 2014, 2014.

[19] S. M. Abd El-kader y B. M. Mohammad El-Basioni, «Precision farming solution in Egypt using the wireless sensor network technology,» Egyptian Informatics Journal, vol. 14, nº 3, p. 221-233, 2013.

[20] Aqeel-ur-Rehman, A. Z. Abbasi, N. Islam y Z. Ahmed Shaikh, «A review of wireless sensors and networks' applications in agriculture,» Computer Standards \& Interfaces, vol. 36, n 2, p. 263-270, 2014.

[21] B. M. Mohammad El-Basioni, S. M. Abd El-kader y M. Abdelmonim Fakhreldin, «Smart Home Design using Wireless Sensor Network and Biometric Technologies,» International Journal of Application or Innovation in Engineering \& Management (IJAIEM), vol. 2, $\mathrm{n}^{\circ}$ 3, 2013.

[22] N. Mitton, S. Papavassiliou, A. Puliafito y K. S. Trivedi, «Combining Cloud and sensors in a smart city environment,» EURASIP Journal on Wireless Communications and Networking, pp. 1-10, 2012.

[23] J. Chinrungrueng, U. Sunantachaikul y S. Triamlumlerd, «Smart Parking: An Application of Optical Wireless Sensor Network,» de International Symposium on Applications and the Internet Workshops, 2007. SAINT Workshops 2007, Hiroshima, 2007.

[24] N. Xu, S. Rangwala, K. Kant Chintalapudi, D. Ganesan, A. Broad, R. Govindan y D. Estrin, «A wireless sensor network For structural monitoring,» de SenSys '04 Proceedings of the 2nd international conference on Embedded networked sensor systems, New York, USA, 2004.

[25] S. Pakzad, G. Fenves, S. Kim y D. Culler, «Design and Implementation of Scalable Wireless Sensor Network for Structural Monitoring,» Journal of Infrastructure Systems, vol. 14, 2008.

[26] T. Torfs , T. Sterken, S. Brebels, J. Santana, R. van den Hoven, V. Spiering, N. Bertsch, D. Trapani y D. Zonta, «Low Power Wireless Sensor Network for Building Monitoring,» IEEE Sensors Journal, vol. 13, n 3, pp. 909 915, 2013.

[27] M. Li y H.-J. Lin, «Design and Implementation of Smart Home Control Systems Based on Wireless Sensor Networks and Power Line Communications,»IEEE Transactions on Industrial Electronics, vol. 62, n 7, pp. 4430 - 4442, 2015. 
[28] J. Yang, J. Zhou, Z. Lv, W. Wei y H. Song, «A Real-Time Monitoring System of Industry Carbon Monoxide Based on Wireless Sensor Networks,» Sensors, vol. 15, n 11, 2015.

[29] J. Wang, H. Wang, J. He, L. Li, M. Shen, X. Tan, H. Min y L. Zheng, «Wireless sensor network for real-time perishable food supply chain management,» Computers and Electronics in Agriculture, vol. 110, p. 196-207, 2015.

[30] F. M. Al-Turjman, H. S. Hassanein y M. A. Ibnkahlab, «Efficient deployment of wireless sensor networks targeting environment monitoring applications,» Computer Communications, vol. 36, $\mathrm{n}^{\circ}$ 1, p. 135-148, 2013.

[31] M. Pejanović Đurišić, Z. Tafa, G. Dimić y V. Milutinović, «A survey of military applications of wireless sensor networks,» de Mediterranean Conference on Embedded Computing (MECO), Bar, 2012.

[32] X. Yu, P. Weller y K. Grattan, «A WSN healthcare monitoring system for elderly people in geriatric facilities,» Digital Healthcare Empowering Europeans, 2015.

[33] L. Catarinucci, D. De Donno, L. Mainetti, L. Palano y L. Patrono, «Integration of UHF RFID and WSN technologies in healthcare systems,» de RFID Technology and Applications Conference (RFID-TA), IEEE, Tampere, 2014.

[34] A. Somov, A. Baranov, D. Spirjakin, A. Spirjakin, V. Sleptsov y R. Passerone, «Deployment and evaluation of a wireless sensor network for methane leak detection,» Sensors and Actuators A: Physical, vol. 202, p. 217-225, 2013.

[35] X. Niu, X. Huang, Z. Zhao, Y. Zhang, C. Huang y L. Cui, «The Design and Evaluation of a Wireless Sensor Network for Mine Safety Monitoring,» de IEEE GLOBECOM 2007 - IEEE Global Telecommunications Conference, Washington, DC, 2007.

[36] International Telecommunication Union, «Series Y: Global Information Infrastructure, Internet Protocol Aspects and Next-Generation Networks (Y.2060),» Telecommunication Standardization Sector of ITU, 2012.

[37] F. Zhang, «Research on Applications of Internet of Things in Agriculture,» de Informatics and Management Science VI, 2013, pp. 69-75.

[38] L. Sánchez, L. Muñoz, J. A. Galache, P. Sotres, J. R. Santana, V. Gutierrez, R. Ramdhany, A. Gluhak, S. Krco, E. Theodoridis y D. Pfisterer, «SmartSantander: IoT experimentation over a smart city testbed,» Computer Networks, vol. 61, p. 217-238, 2014.

[39] L. Ding, Z. C., Q. Deng, H. B. Luo, Y. X.W., Y. Q. Ni y P. Guo, «Real-time safety early warning system for cross passage construction in Yangtze Riverbed Metro Tunnel based on the internet of things,» Automation in Construction, vol. 36, p. 25-37, 2013.

[40] R. Piyare, «Internet of Things: Ubiquitous Home Control and Monitoring System using Android based Smart Phone,» International Journal of Internet of Things, vol. 2, $\mathrm{n}^{\circ}$ 1, pp. 5-11, 2013.

[41] L. Da Xu , W. He y S. Li, «Internet of Things in Industries: A Survey,» IEEE Transactions on Industrial Informatics, vol. 10, $\mathrm{n}^{\circ}$ 4, pp. 2233 - 2243, 2014.

[42] J. Jiang y K. Su, «Management Platform Architecture of Modern Tobacco Logistics Based on Internet of Things Technologies,» de LISS 2012, 2013, pp. 1403-1409.

[43] F. Lao y G. Xin Li, «The Design and Implementation of Crop Growing Environment Monitoring System Based on the Internet of Things,» Advanced Materials Research, Vols. \%1 de \%2912-914, pp. 1440-1443, 2014.

[44] Z. Kang-yi, A. Zhi-gang, T. Chang-chun, B. Heng-guang y F. Go, «Application of Internet of Things in Military,» Internet of Things Technologies, 2012.

[45] F. Hu, D. Xie y S. Shen, «On the Application of the Internet of Things in the Field of Medical and Health Care,» de Green Computing and Communications (GreenCom), 2013 IEEE and Internet of Things (iThings/CPSCom), IEEE International Conference on and IEEE Cyber, Physical and Social Computing, Beijing, 2013.

[46] C. Du y S. Zhu, «Research on Urban Public Safety Emergency Management Early Warning System based on Technologies for the Internet of Things,» Procedia Engineering, vol. 45, pp. 748-754, 2012.

[47] J. Yick, B. Mukherjee y D. Ghosal, «Wireless sensor network survey,» Computer Networks, vol. 52, p. 22922330, 2008

[48] Gartner, «The Internet of Things and Related Definitions,» 2014.

[49] GSMA, «Understanding the Internet of Things (IoT),» GSMA Head Office, New Fetter Lane, London, UK, 2014.

[50] CERP-IoT, «Internet of Thing. Strategic Research Roadmap,» 2009.

[51] CASAGRAS, «RFID and tje Inclusive Model for the Internet of Things,» 2009.

[52] S. Hadim y N. Mohamed, «Middleware for Wireless Sensor Networks: A Survey,» de 1st International Conference on Communication Systems Software \& Middleware, New Delhi, 2006.

[53] S. Hadim y N. Mohamed, «Middleware: Middleware Challenges and Approaches for Wireless Sensor Networks,» IEEE Distributed Systems Online , vol. 7, n 3, pp. 1-23, 2006.

[54] E. Borgia, «The Internet of Things vision: Key features, applications and open issues,» Computer Communications, p. 1-31, 2014.

[55] IoT World Forum, «IoT Reference Model Whitepaper,» 2014. 
[56] Industrial Internet Consortium, «Industrial Internet Reference Architecture,» 2015.

[57] International Electrotechnical Commussion, «Internet of Thing: Wireless Sensor Networks,» Wireless Sensor Networks Project Team, Geneva, Switzerland.

[58] I. Lee y K. Lee, «TheInternetofThings(IoT):Applications, investments, and challenges for enterprises,» Business Horizons, vol. 58, p. 431-440, 2015.

[59] Bluetooth Inc, «Bluetooth core specification,» Bluetooth SIG, Inc., 2016. [En línea]. Available: https://www.bluetooth.com/specifications/bluetooth-core-specification.

[60] 3GPP, «General Packet Radio Service (GPRS); Service description; Stage 2,» The 3rd Generation Partnership Project (3GPP), 2016. [En línea]. Available: http://www.3gpp.org/dynareport/23060.htm.

[61] P. Thubert, A. Brandt, J. Hui, R. Kelsey, P. Levis, K. Pister, R. Struik, J. Vasseur y R. Alexander, «RFC 6550: RPL: IPv6 Routing Protocol for Low-Power and Lossy Networks,» Internet Engineering Task Force (IETF) , 2012.

[62] N. Kushalnagar, G. Montenegro y C. Schumacher, «RFC 4919: IPv6 over Low-Power Wireless Personal Area Networks (6LoWPANs): Overview, Assumptions, Problem Statement, and Goals,» Network Working Group , 2007.

[63] W3C, «REST,» 2011. [En línea]. Available: https://www.w3.org/2001/sw/wiki/REST.

[64] Z. Shelby, K. Hartke y C. Bormann, «RFC 7250: The Constrained Application Protocol (CoAP),» Internet Engineering Task Force (IETF), 2014.

[65] International Organization for Standardization, «ISO/IEC 20922:2016 - Information technology -- Message Queuing Telemetry Transport (MQTT) v3.1.1,» 2010.

[66] P. Saint-Andre, «RFC 6120: Extensible Messaging and Presence Protocol (XMPP): Core,» Internet Engineering Task Force (IETF), 2011.

[67] W3C, «Extensible Markup Language (XML) 1.0 (Fifth Edition),» 2008.

[68] T. Bray, «RFC 7159: The JavaScript Object Notation (JSON) Data Interchange Format,» Internet Engineering Task Force (IETF) , 2014.

[69] K. Lingaraj, R. V. Biradar y V. C. \& Patil, «A Survey on Middleware Challenges and Approaches for Wireless Sensor Networks,» de Computational Intelligence and Communication Networks (CICN), 2015 International Conference on, 2015.

[70] B. Bhuyan, H. K. Deva Sarma y N. Sarma, «A Survey on Middleware for Wireless Sensor Networks,» Journal of Wireless Networking and Communications, vol. 4, n 1, pp. 7-17, 2014.

[71] M. T. Wang, J. S. Wei, Y. H. Pan y Z. \& Wei, «Study on Data Fusion Techniques in Wireless Sensor Networks,» de 6th International Asia Conference on Industrial Engineering and Management Innovation, 2016.

[72] A. Tripathi, S. Gupta y B. Chourasiya, «Survey on Data Aggregation Techniques for Wireless Sensor Networks, » de International Journal of Advanced Research in Computer and Communication Engineering, 2014.

[73] P. Jesus, C. Baquero y P. S. Almeida, «A Survey of Distributed Data Aggregation Algorithms,» IEEE Communications Surveys \& Tutorials, vol. 17, $\mathrm{n}^{\circ}$ 1, pp. $381-404,2014$.

[74] S. Deering y R. Hinden, «RFC 2460: Internet Protocol, Version 6 (IPv6) Specification,» Network Working Group, 1998.

[75] B. Bosančić, «Information in the Knowledge Acquisition Process,» Journal of Documentation, 2016.

[76] J. A. Manrique, J. S. Rueda-Rueda, and J. M. T. Portocarrero, "Contrasting Internet of Things and Wireless Sensor Network from a conceptual overview," in Internet of Things (iThings) and IEEE Green Computing and Communications (GreenCom) and IEEE Cyber, Physical and Social Computing (CPSCom) and IEEE Smart Data (SmartData), 2016 IEEE International Conference on, 2016, pp. 252-257.

[77] H. B. Pandya y T. A. Champaneria, «Internet of things: Survey and case studies,» de International Conference on Electrical, Electronics, Signals, Communication and Optimization (EESCO), 2015, Visakhapatnam, 2015.

[78] IEEE, «Towards a Definition of the Internet of Things (IoT),» IEEE Internet of Things, 2015.

[79] CERP-IoT: Cluster of European Research Projects on the Internet of Things, «Vision and Challenges for Realising the Internet of Things,» European Commission - Information Society and Media DG , Brussels, 2010.

[80] Acemap Inc, «Acemap, Academic Paper Recommendation,» Shanghai Jiao Tong University, 2016. [En línea]. Available: http://acemap.situ.edu.cn/.

[81] Acemap Inc, «Development and Trends on Wireless Sensor Network,» Shanghai Jiao Tong University, Julio 2016. [En línea]. Available: http://acemap.sjtu.edu.cn/topic/topicpage?topicID=017737EA. [Último acceso: Julio 2016].

[82] Acemap Inc., «Development and Trends on Internet of Things,» Shanghai Jiao Tong University, Julio 2016. [En línea]. Available: http://acemap.sjtu.edu.cn/topic/topicpage?topicID=04E11757. [Último acceso: Julio 2016]. 


\section{Sobre los autores}

Johan S. Rueda R.

Asistente investigador del Centro de Excelencia y Apropiación en Internet de las Cosas, CEA-IoT. Estudiante de maestría en telemática en la Universidad Autónoma de Bucaramanga - UNAB. Especialista en Auditoria de Sistemas (2016) e Ingeniero de Sistemas de la Universidad Francisco de Paula Santander - Ocaña, (2015). Sus intereses investigativos incluyen seguridad de la información, ciberseguridad, auditoria de TI e IoT.

\section{Jesús M. Talavera P.}

Integrante del Laboratorio de investigación en fusión de datos, información y conocimiento de la Universidad Federal de Río de Janeiro, Brasil. Doctor en Informática, Universidad Federal de Rio de Janeiro (2016), RJ-Brasil. Magister en Ciencia de la Computación, Universidad Federal de Sao Carlos (2010), SP-Brasil. Ingeniero de Sistemas, Universidad Católica de Santa María (2006), Perú. Sus intereses investigativos incluyen sistemas middleware para WSN, IoT y arquitecturas de software. 